\title{
Sistema Heterogéneo para la Monitorización de la Actividad Diaria en el Hogar y el Bienestar de Personas Mayores
}

\author{
Francisco Miguel Calatrava Nicolás \\ ETSII, Universidad Politécnica de Cartagena. Calle Dr. Fleming, s/n, Cartagena \\ francisco.calatrava@edu.upct.es
Francisco José Ortiz Zaragoza ${ }^{1}$, José Alfonso Vera Repullo ${ }^{1}$, Joaquín Roca González ${ }^{1}$, Manuel Jiménez Buendía $^{1}$, Óscar Martínez Mozos ${ }^{2}$, ${ }^{1}$ ETSII, Universidad Politécnica de Cartagena. Calle Dr. Fleming, s/n, Cartagena
${ }^{2}$ AASS, Örebro University, Suecia
francisco.ortiz@upct.es, jose.vera@upct.es, jroca.gonzalez@upct.es, manuel.jimenez@upct.es, \\ oscar.mozos@oru.se
}

\begin{abstract}
Resumen
En este artículo se presenta el diseño de un sistema heterogéneo cuya finalidad es la de cuidar la salud y el bienestar de las personas mayores que viven solas en su hogar. Se intenta seguir la iniciativa del programa europeo AAL (Ambient Assisted Living) Dicho sistema se encuentra formado por un dispositivo robótico móvil, un conjunto de sensores domóticos de bajo coste, un dispositivo médico tipo pulsera de actividad y una aplicación de Android para el estudio del estado anímico. El sistema ha sido integrado haciendo uso de ROS (Robot Operating System), de tecnologías IoT (Internet of Things) tales como Node-RED y la plataforma domótica Home-Assistant. Este sistema heterogéneo se desarrolla en la actualidad en un proyecto nacional Retos de la Sociedad.
\end{abstract}

Palabras clave: Robótica asistencial, envejecimiento, Ambient Assisted Living (AAL), Node-RED, IoT, interoperablilidad, sistemas heterogéneos, ROS, Smart Home.

\section{INTRODUCCIÓN}

Los avances científicos y tecnológicos han traído consigo mejoras en la forma y calidad de vida de las personas, haciéndose notar en la longevidad. Sin ir más lejos, a pesar del declive de 1.6 años acontecido debido a la pandemia de la COVID 19, España sigue encabezando las listas en cuanto a longevidad en Europa, pasando, en términos totales, de 84 a 82.4 años [1]. Suponiendo el fin de la vida laboral a los 65 años, se pueden contabilizar, aproximadamente, 17.2 años de merecida jubilación. Desgraciadamente, a la par que pasa el tiempo, la salud suele empeorar, dejando, normalmente, lo peor para el final. Por otro lado, también existe el problema de la inclinación cada vez mayor a vivir esa etapa en soledad.

Tal y como se puede observar en la Figura 1, la tendencia a vivir en soledad para mayores de 65 años sigue siendo ascendente en los últimos diez años. Así pues, y centrándonos en la situación española, se puede concluir que se vive más tiempo, la salud se ve más afectada en los últimos años de vida y, además, se está incrementando la tendencia de vivir en soledad. Estos tres factores (esperanza de vida, deterioro de la salud y soledad) constituyen la motivación del proyecto coordinado HIMTAE (Heterogeneous Intelligent Multi-Robot Team for Assistance of Elderly People), del programa Retos de la Sociedad del Ministerio (2019-2021), en el que colaboran diferentes investigadores de la Universidad Politécnica de Cartagena, la Universidad de Murcia, la Universidad Carlos III de Madrid y la Universidad de Örebro en Suecia.

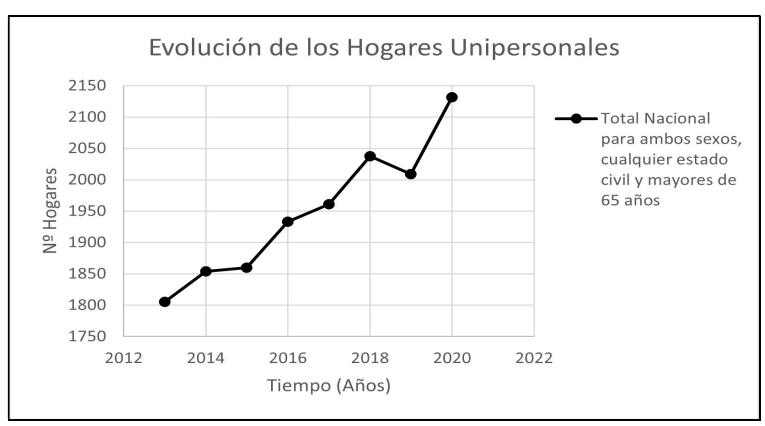

Figura 1: Evolución de los hogares unipersonales en España teniendo en cuenta la población nacional para ambos sexos, cualquier estado civil y mayores de 65 años (Datos obtenido de [2]).

Este artículo se centra en la exposición del sistema que engloba el subproyecto Robwell (Robotic-based Well-Being Monitoring and Coaching for Elderly People during Daily Life Activities) cuya principal 
misión es la de obtener información acerca de las rutinas y parámetros fisiológicos del usuario, con la finalidad de realizar una estimación del estado anímico para que el robot móvil ofrezca diferentes actividades con el objetivo de mejorarlo.

Se trata de un sistema heterogéneo que incluye elementos de diferente naturaleza como son la robótica, la domótica y la inteligencia artificial orientada al análisis del estado anímico del usuario. El sistema robótico integrado con inteligencia ambiental se enmarca en el campo de la robótica asistencial, siendo capaz de interactuar con el usuario y de actuar en función de su estado anímico de manera similar al BotCare de Samsung [3] o al robot Rassel de la UPV [4], que, aunque no es un robot móvil, sí que se centra en la interacción y el reconocimiento del estado anímico. El ámbito de domótica y smart home orientado al bienestar y a la salud de las personas mayores ha tenido un creciente interés en los últimos años. Sistemas como los que se muestran en el proyecto PROSAFE [5], TigerPlace [6] o SMARTA [7] muestran la evolución desde el proyecto de 1996 de Alex Pentland [8] que fue de los primeros que se aproximó a la idea de smart home que se tiene hoy en día. Por otro lado, teniendo en cuenta que el sistema presentado en este proyecto se centra, no solo en la parte de domótica, sino también en la integración, conviene mencionar proyectos como SEAL [9] o el presentado en [10], en los que también se usa ROS como middleware. En cuanto al reconocimiento de estado anímico, cabe destacar que este campo ha ganado mucha importancia dentro del mundo de la inteligencia artificial. Estudios de laboratorio como [11] y [12] han mostrado buenos resultados. Sin embargo, llevar este sistema a la vida real se hace complicado por la cantidad de variables del día a día y por la incomodidad de los sensores de laboratorio. El sistema propuesto en este proyecto pretende llevar el estudio al ambiente cotidiano del hogar mediante una aplicación Android usando EMAs (Ecological Momentary Assessments), que permiten la evaluación del estado de ánimo con 2 preguntas acerca del nivel de felicidad y actividad del usuario, tal y como se propone en un trabajo previo del que parte el equipo investigador del proyecto Robwell [13].

\section{SISTEMA PROPUESTO}

El sistema propuesto se engloba dentro de la tipología de sistema heterogéneo debido a su diversidad. A continuación, se nombran y resumen brevemente los componentes del sistema para luego explicarlos en profundidad.

- Plataforma robótica. Deberá navegar de manera autónoma por la casa para atender a las necesidades del usuario o para sugerirle alguna actividad según su estado anímico.

- Ecosistema de sensores domóticos. Se trata de un conjunto de sensores tanto comerciales como de diseño propio para monitorizar el estilo de vida del usuario.

- Dispositivo médico Empatica E4. Permitirá la monitorización de ciertas variables fisiológicas como son el BVP (Blood Volume Pulse), EDA (Electrodermal Activity), la temperatura cutánea, el HR (Heart Rate), el IBI (Interbeat Interval) y la aceleración tanto en el eje $\mathrm{X}$, como $\mathrm{Y}$ como Z.

- Aplicación Android. Para poder hacer un estudio psicológico y poder relacionar las características de la información obtenida de la Empatica E4 con el estado anímico del usuario, se ha desarrollado una aplicación Android en la que se podrá responder a preguntas básicas sobre actividad/bienestar.

En la Figura 2 se pueden observar algunos de los elementos del sistema.

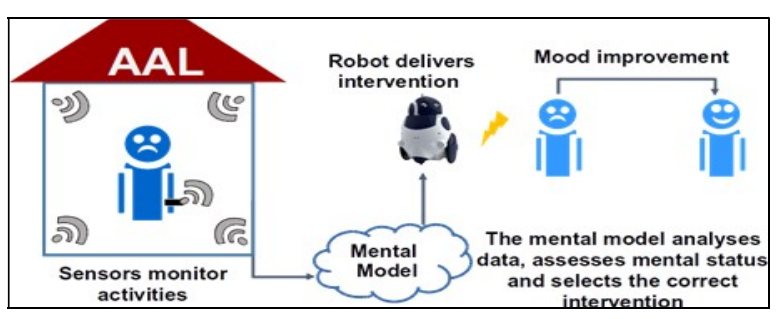

Figura 2: Sistema general del subproyecto Robwell.

Uno de los principales problemas de los sistemas heterogéneos es, precisamente, el reto de integrar los elementos que intervienen en el mismo. El principal problema en este proyecto viene de la mano del sistema domótico. Tal y como se ha mencionado, se pretende ser lo menos intrusivo posible y se buscan, siempre que sea posible, soluciones comerciales de bajo coste. Por ello, uno de los principales retos de este proyecto es diseñar y desarrollar un componente hardware/software intermedio encargado de hacer transparente la información entre los diferentes elementos del sistema. Para llevar a cabo esta idea se ha utilizado ROS y tecnologías de IoT (Internet of Things).

\subsection{PLATAFORMA ROBÓTICA}

Para la plataforma robótica se ha optado por la solución comercial Turtlebot II [14]. Se trata de un robot móvil de tipo diferencial con dos ruedas de apoyo situadas en situación romboidal. La base móvil sobre la que se monta el resto de la plataforma robótica es la IClebo de Kobuki. Dicha base ofrece la posibilidad de montar encima plataformas según una 
estructura modular para incorporar el hardware extra necesario como CPU, LIDAR, etc. Para conseguir una interfaz con el usuario que integre lenguaje natural además de la interacción visual, en este proyecto se monta en la última de sus plataformas un altavoz inteligente tipo Alexa 10.

Para llevar a cabo la navegación autónoma del robot dentro del hogar evitando los objetos tanto estáticos como dinámicos, el robot dispone de un sensor LIDAR Hokuyo UST-10LX que le permite obtener información precisa del entorno para el mapeo y navegación. Para este propósito, en una primera aproximación se ha usado el stack de navegación de ROS configurándolo para el uso del Hokuyo UST10LX. No obstante, se tiene previsto implementar métodos de navegación semántica para mejorar la fiabilidad de esta navegación autónoma.

\subsection{ECOSISTEMA DE SENSORES}

Esta parte del sistema incluye todos los sensores domóticos comerciales y de desarrollo propio que aportarán información acerca del comportamiento y los hábitos del usuario. En la Figura 3 se puede observar el reparto en la casa de los sensores domóticos básicos comerciales a falta del sensor de inundación y la puerta universal de infrarrojos.

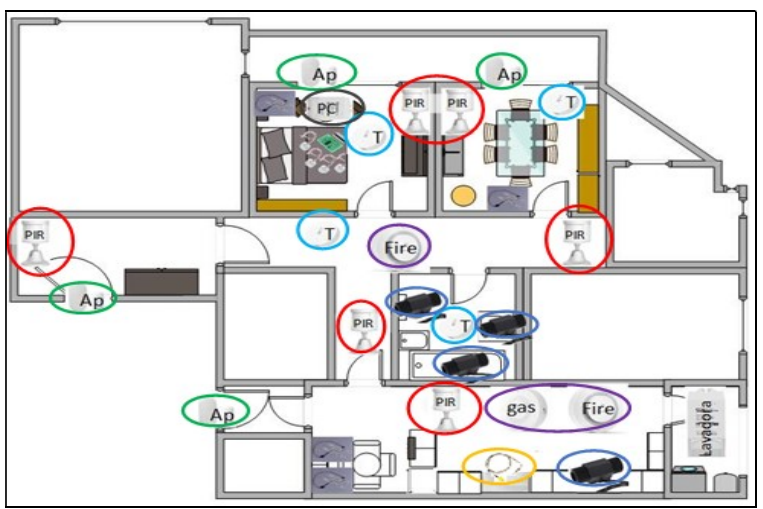

Figura 3: Distribución de los sensores domóticos $(\mathrm{AP}=$ Apertura/verde, $\mathrm{PIR}=$ Presencia/rojo, $\mathrm{T}=$ Temperatura/azul claro, $\mathrm{PC}=$ Consumo Eléctrico/negro, gas $=$ gas natural/violeta, fire $=$ fuego/violeta, azul oscuro= sensor de flujo, amarillo=termopar)

Tabla 1: Sensores del sistema.

\begin{tabular}{|c|c|c|}
\hline $\begin{array}{c}\text { Nombre } \\
\text { Sensor }\end{array}$ & Modelo & Descripción \\
\hline $\begin{array}{c}\text { Mi Motion } \\
\text { Sensor }\end{array}$ & YTC4041GL & $\begin{array}{c}\text { Sensor PIR de Xiaomi } \\
\text { (PIR) }\end{array}$ \\
\hline $\begin{array}{c}\text { Temp/Hum } \\
\text { Aqara }\end{array}$ & WSDCGQ11LM & $\begin{array}{c}\text { Sensor de temperatura y } \\
\text { humedad de Aqara (T) }\end{array}$ \\
\hline $\begin{array}{c}\text { Mi } \\
\text { Window/Door } \\
\text { Sensor }\end{array}$ & YTC4039GL & $\begin{array}{c}\text { Sensor de puerta y } \\
\text { ventana de Xiaomi (Ap) }\end{array}$ \\
\hline
\end{tabular}

\begin{tabular}{|c|c|c|}
\hline $\begin{array}{l}\text { Detector Gas } \\
\text { Heiman }\end{array}$ & HS1CG & $\begin{array}{l}\text { Detector de gas natural } \\
\text { de Heiman (gas) }\end{array}$ \\
\hline $\begin{array}{c}\text { Detector de } \\
\text { Inundación } \\
\text { Aqara }\end{array}$ & SJCGQ11LM & $\begin{array}{c}\text { Detector de inundación } \\
\text { de Aqara }\end{array}$ \\
\hline $\begin{array}{c}\text { Detector de } \\
\text { Humo Xiaomi }\end{array}$ & $\begin{array}{c}\text { JTYJ-GD- } \\
\text { 03MI/BB }\end{array}$ & $\begin{array}{c}\text { Detector de humo y } \\
\text { fuego de Xiaomi (fire) }\end{array}$ \\
\hline Sonoff POW & IM171130001 & $\begin{array}{c}\text { Medida de consumos } \\
\text { (PC) }\end{array}$ \\
\hline $\begin{array}{l}\text { Broadlink } \\
\text { RM-Mini3 }\end{array}$ & RM-MINI3 & $\begin{array}{c}\text { Puerta universal para } \\
\text { infrarrojos }\end{array}$ \\
\hline $\begin{array}{l}\text { Sensor de } \\
\text { flujo }\end{array}$ & $\begin{array}{l}\text { Desarrollo } \\
\text { propio }\end{array}$ & $\begin{array}{c}\text { Sensor para la } \\
\text { monitorización del uso } \\
\text { de los grifos del aseo y } \\
\text { cocina }\end{array}$ \\
\hline $\begin{array}{l}\text { Sensor de } \\
\text { sentado }\end{array}$ & $\begin{array}{l}\text { Desarrollo } \\
\text { propio }\end{array}$ & $\begin{array}{c}\text { Sensor para la } \\
\text { monitorización del } \\
\text { tiempo que pasa el } \\
\text { usuario sentado }\end{array}$ \\
\hline $\begin{array}{l}\text { Sensor de } \\
\text { pesado de } \\
\text { cama }\end{array}$ & $\begin{array}{l}\text { Desarrollo } \\
\text { propio }\end{array}$ & $\begin{array}{c}\text { Sensor para la } \\
\text { monitorización del peso } \\
\text { del usuario y del tiempo } \\
\text { que pasa en cama }\end{array}$ \\
\hline $\begin{array}{l}\text { Sensor de } \\
\text { consumos } \\
\text { grandes }\end{array}$ & $\begin{array}{l}\text { Desarrollo } \\
\text { propio }\end{array}$ & $\begin{array}{c}\text { Sensor para dispositivos } \\
\text { de gran consumo de } \\
\text { casa. Se usará el } \\
\text { PZEM-004T. }\end{array}$ \\
\hline $\begin{array}{c}\text { Termopar } \\
\text { vitro/fogones }\end{array}$ & $\begin{array}{l}\text { Desarrollo } \\
\text { propio }\end{array}$ & $\begin{array}{c}\text { Sensor para monitorizar } \\
\text { el uso de la vitro o los } \\
\text { fogones }\end{array}$ \\
\hline $\begin{array}{l}\text { Sensor } \\
\text { teléfono fijo }\end{array}$ & $\begin{array}{l}\text { Desarrollo } \\
\text { propio }\end{array}$ & $\begin{array}{l}\text { Sensor para monitorizar } \\
\text { la interacción social a } \\
\text { través del teléfono fijo }\end{array}$ \\
\hline $\begin{array}{c}\text { Sistema de } \\
\text { localización }\end{array}$ & $\begin{array}{l}\text { Desarrollo } \\
\text { propio }\end{array}$ & $\begin{array}{l}\text { Sistema de localización } \\
\text { en casa con decaWave }\end{array}$ \\
\hline
\end{tabular}

Como se puede ver en la Tabla 1, se han propuesto una gran cantidad de elementos para monitorizar la actividad de usuario y el estado de la vivienda. Los 8 primeros de la tabla son dispositivos comerciales y los restantes son de diseño propio. La mayor parte de los sensores comerciales usan el protocolo Zigbee, por tanto, para poder realizar una gestión de los mismos de manera local desde Home Assistant, se están usando 2 puertas de enlace genéricas: cc2531 y Conbee II.

Por otro lado, para los sensores de diseño propio, se utiliza un microcontrolador de bajo coste ESP32 [15] para publicar la información de los mismo mediante el protocolo de comunicación MQTT a un broker Mosquitto montado sobre la misma Raspberry pi que Home Assistant.

\subsection{EMPATICA E4 Y APLICACIÓN MÓVIL}

La Empatica E4 es un dispositivo médico que tiene 2 modos de funcionamiento. El que se va a usar para el estudio es el llamado modo streaming. Haciendo uso de la aplicación oficial de Empatica E4 (E4 RealTime), se establece la conexión entre el dispositivo médico y el smartphone mediante BLE (Bluetooth Low Energy) y se comienza a tomar datos. Una vez se termine dicha toma de datos, ya sea por desconexión no intencionada del dispositivo o por desconexión intencionada, los datos se subirán a 
la nube propia de Empatica E4, donde serán accesibles y descargables. En la Tabla 2 se pueden observar las principales características de los sensores de la Empatica E4.

Tabla 2: Frecuencia de muestreo de los sensores de Empatica E4.

\begin{tabular}{|c|c|}
\hline Sensor & Frecuencia Muestreo \\
\hline BVP & $64 \mathrm{~Hz}$ \\
Acelerómetro & $32 \mathrm{~Hz}$ \\
Temperatura Cutánea & $4 \mathrm{~Hz}$ \\
EDA & $4 \mathrm{~Hz}$ \\
\hline
\end{tabular}

En cuanto a la aplicación Android desarrollada para el estudio, tiene como principal requisito permitir recoger información de cómo se siente el usuario en cuanto a su nivel de felicidad y actividad de la forma más simple posible. Se hace con el objetivo de poder contrastar sus respuestas con los datos fisiológicos recogidos con la Empatica E4 en los instantes pertinentes y, después de entrenar el algoritmo de machine learning con dichos datos, llegar a poder estimar el estado de ánimo del usuario sin necesidad de tener que responder a los cuestionarios [13]. La imposición de diseño inicial fue la de crear un sistema móvil-Empatica E4 que se pudiera entregar al sujeto de la prueba como si fuera un pack y con todas las configuraciones pertinentes hechas. Se decidió así debido a que la aplicación desarrollada necesita permisos para el acceso a las notificaciones del smartphone y se llegó a la conclusión de que instalar una aplicación de estas características en un móvil personal podría considerarse intrusivo.

La aplicación dispone de 2 modos: un modo usuario y un modo administrador. Dentro del modo usuario solo se permitirá contestar a un cuestionario en el que el sujeto de la prueba deberá responder como de activo y de feliz se siente en una escala de 0 a 4 . El modo administrador dispone de un menú principal en el que se puede acceder a la pantalla del cuestionario y a la configuración de la aplicación. Dentro de la configuración se pueden establecer las horas a las que se quiere que se dispare una notificación que avise al sujeto de la prueba de que tiene que realizar el cuestionario. Por otro lado, la aplicación permite inicializar una clave de usuario para cada uno de los sujetos. Así se evita registrar datos personales de los usuarios. Cada una de las claves serán proporcionadas por el investigador.

Como se puede observar en la Figura 4, en el menú se tienen más opciones de las que se han explicado. Esto se debe a que, actualmente, la aplicación sigue en desarrollo.

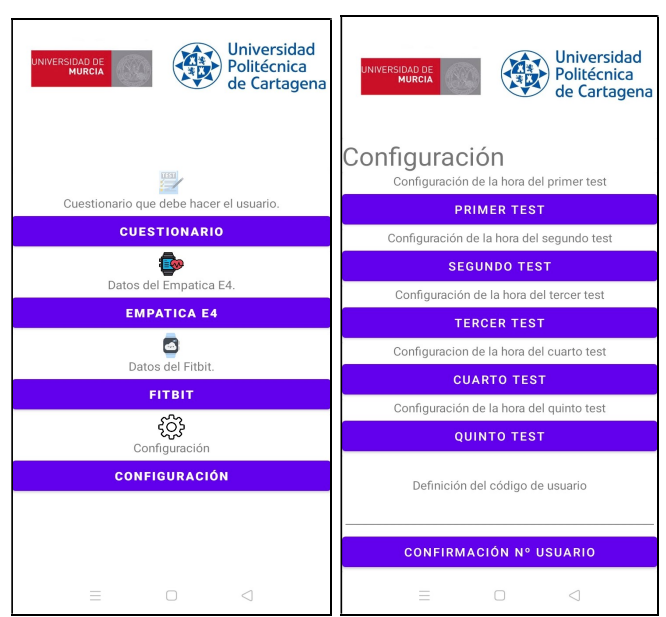

Figura 4. Opciones del modo administrador.

La idea final es no necesitar la aplicación de Empatica E4 RealTime, sino que desde la aplicación desarrollada se publiquen los datos de los sensores en el broker Mosquitto que va instalado sobre Home Assistant. De esta manera será accesible desde todo el sistema. En cuanto al apartado Fitbit de la aplicación, se ha añadido debido a que se tiene planeado usar una pulsera de actividad Fitbit Sense para en proyecto. El objetivo final es validar los datos de la Fitbit Sense con los datos de la Empatica E4 y, de esta manera, prescindir de la Empatica E4 como componente del sistema final. El principal motivo por el que se tiene previsto prescindir de la Empatica E4 es el precio.

Como se puede observar en la Figura 5, el modo usuario solamente dispone de la posibilidad de realizar el cuestionario. Para la selección del nivel de felicidad y de actividad se dispone de 2 barras discretas con 5 niveles (0-4).

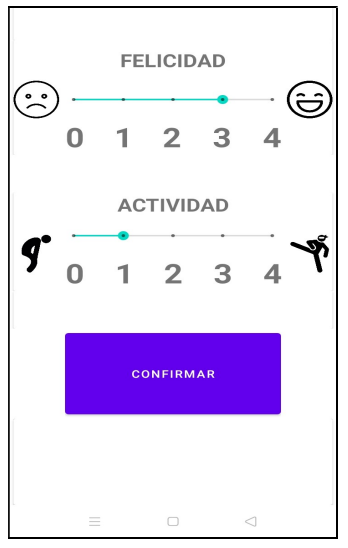

Figura 5: Opción del modo usuario.

La información total que se obtiene de esta aplicación es la siguiente: 
- Información actividad/felicidad. Se guarda el resultado del cuestionario junto con el timestamp de la realización del mismo.

- Información de reacción del usuario. Se guarda el timestamp del momento en el que se lanza la notificación correspondiente al cuestionario y el timestamp del momento en el que el usuario pulsa la notificación para acceder a la pantalla del cuestionario.

- Información de las horas programadas de los cuestionarios. Se guardan las horas definidas a las que deben lanzarse las notificaciones de la realización del cuestionario y todas las modificaciones que haga el usuario.

Toda esta información se guardará en archivos locales de la aplicación y en una hoja de cálculo en la nube (Google Sheet).

\section{INTEGRACIÓN DE ELEMENTOS}

Para la integración de todos los elementos del sistema robótico, sensores domóticos e información fisiológica se ha decidido usar como middleware ROS ya que entre sus paquetes ofrecen muchas opciones de conectividad. Por otra parte, el hecho de que permita organizar la arquitectura en nodos siendo multilenguaje y siguiendo la política de publicador/suscriptor aporta flexibilidad y facilidad de entendimiento en el diseño.

Hasta el momento se han diseñado y validado los caminos de integración entre los diferentes elementos del sistema heterogéneo y ROS. Actualmente se está trabajando en la implementación de la arquitectura software que va a ir sobre ROS para aportar al sistema heterogéneo interoperabilidad $y$ transparencia entre los componentes.

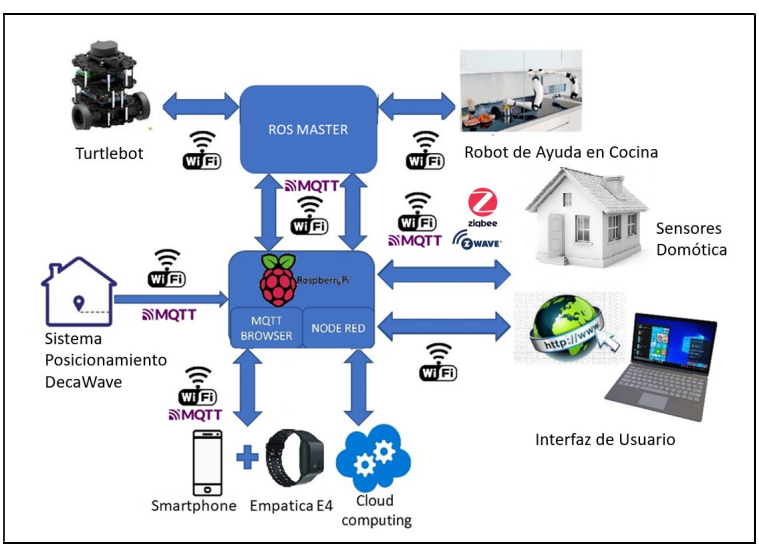

Figura 6: Esquema general de integración.

En la Figura 6 se puede observar un esquema general del sistema. El sistema para identificar la posición del usuario dentro de la casa está aún en desarrollo. En cuanto al robot de ayuda a la cocina, no se encuentra dentro del subproyecto Robwell, sino que del desarrollo del mismo se encarga la universidad Carlos III de Madrid. No obstante, al estar programado con ROS, su integración con la arquitectura global del sistema heterogéneo es igualmente compatible y ha sido validada.

Por otra parte, en la Figura 6 se pueden destacar dos bloques sobre los que se van a montar la mayoría del sistema. Estos bloques son:

- $\quad$ Raspberry Pi. Se trata de una Raspberry Pi 4 de $8 \mathrm{~Gb}$ de memoria RAM a la que se le ha añadido un disco duro SSD de $240 \mathrm{~Gb}$ que va a llevar instalado Home Assistant. Sobre la misma se usará una puerta de enlace genérica zigbee cc2531 y Conbee II, un broker Mosquitto MQTT, un servidor NodeRED y un software de Home Assistant llamado Zigbee2MQTT. De esta manera se podrá obtener información de los sensores domóticos. Además, así se tiene preparado el sistema para recibir información del dispositivo Empatica E4 mediante MQTT cuando la extensión de la aplicación Android esté preparada.

- ROS Master. Se trata de un PC tipo IntelNUC en el que se ha instalado Ubuntu 16.04 con la finalidad de ejecutar sobre el mismo ROS Kinetic. Sobre este PC se ejecutará la lógica y el software que proporcionará interoperabilidad y transparencia entre los diferentes elementos del sistema. Para la integración con Home Assistant se ejecutará el websocket del paquete rosbridge server. De esta manera, se podrá publicar y suscribirse a los topics desde Node-RED. Por otro lado, la integración con otros elementos del sistema que lleven implementado ROS Kinetic es directa.

\section{PRUEBAS, RESULTADOS Y CONCLUSIONES}

Como ya se ha mencionado en el artículo, aunque el sistema se encuentra en una fase avanzada de diseño y desarrollo, todavía no está terminado. La mayoría de las pruebas que se han hecho han sido de integración, de validación individual de los diferentes nodos y de funcionamiento del robot. También se está llevando a cabo en este momento la fase de recogida de datos de diversos usuarios, por eso no se ha incluido en el artículo el procesamiento con machine learning de los mismos. 
Un ejemplo de ello puede ser el funcionamiento de la navegación de la plataforma robótica. Se consiguió adaptar con éxito el stack de navegación de ROS para un mapeo teleoperado y una navegación autónoma con el LIDAR Hokuyo UST-10LX en un entorno real de una casa. En la Figura 7 se puede observar un ejemplo de mapeo.

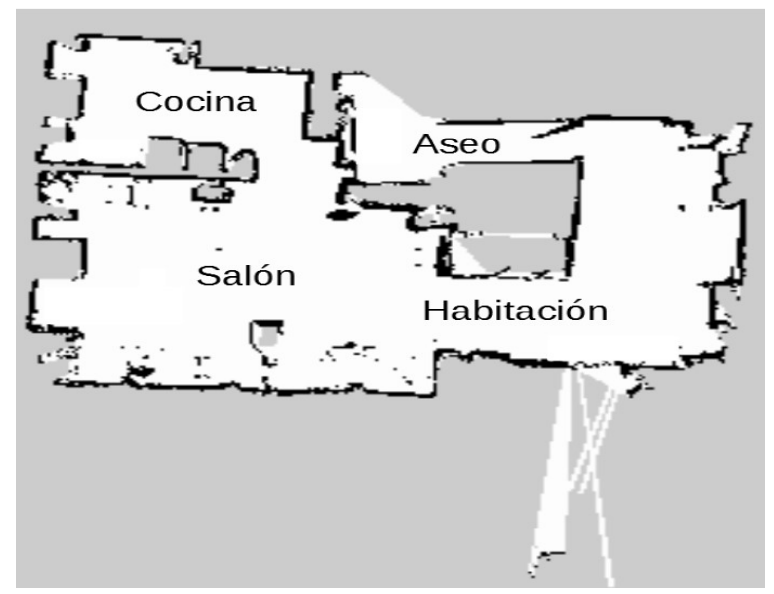

Figura 7: Mapeo con Hokuyo UST-10LX.

Para la prueba de navegación autónoma, se propusieron una serie de puntos de navegación en el entorno hasta los que tendría que navegar en ciertas horas del día. Tras terminar el circuito propuesto, debería volver a la estación de carga ejecutando un algoritmo de autodocking. En la Tabla 3 se pueden observar los puntos de navegación que se propusieron.

Tabla 3: Puntos de navegación ( $\mathrm{H}=$ Habitación, $\mathrm{A}=$ Aseo, $\mathrm{S}=$ Salón, $\mathrm{C}=$ Cocina, $\mathrm{E}=$ Estación).

\begin{tabular}{|c|c|c|c|c|c|c|}
\hline \multicolumn{7}{|c|}{ Desayuno } \\
\hline $\mathrm{H}$ & $\mathrm{A}$ & $\mathrm{S}$ & $\mathrm{C}$ & $\mathrm{A}$ & $\mathrm{S}$ & $\mathrm{E}$ \\
\hline $08: 00$ & $08: 05$ & $08: 15$ & $08: 17$ & $08: 37$ & $08: 45$ & $08: 47$ \\
\hline \multicolumn{6}{|c|}{ Merienda } \\
\hline S & $\mathrm{C}$ & $\mathrm{A}$ & $\mathrm{S}$ & $\mathrm{E}$ \\
\hline $11: 30$ & $11: 33$ & $11: 47$ & $12: 00$ & $12: 02$ \\
\hline \multicolumn{7}{|c|}{ Comida } \\
\hline S & $\mathrm{C}$ & $\mathrm{A}$ & $\mathrm{S}$ & $\mathrm{E}$ \\
\hline $13: 30$ & $13: 33$ & $14: 00$ & $14: 10$ & $14: 12$ \\
\hline \multicolumn{7}{|c|}{ Merienda } \\
\hline S & C & A & S & E \\
\hline $17: 30$ & $17: 32$ & $17: 47$ & $17: 57$ & $18: 00$ \\
\hline \multicolumn{7}{|c|}{ Cena } \\
\hline S & C & A & S & E \\
\hline $20: 30$ & $20: 33$ & $21: 02$ & $21: 10$ & $21: 12$ \\
\hline \multicolumn{7}{|c|}{ Dormir } \\
\hline S & H & A & H & E \\
\hline $22: 30$ & $22: 32$ & $22: 35$ & $22: 45$ & $22: 47$ \\
\hline
\end{tabular}

Para poder realizar un seguimiento de la correcta realización del experimento, se representó el cambio de voltaje de la batería del Turtlebot que ofrece ROS al inicializar el paquete básico del mismo.

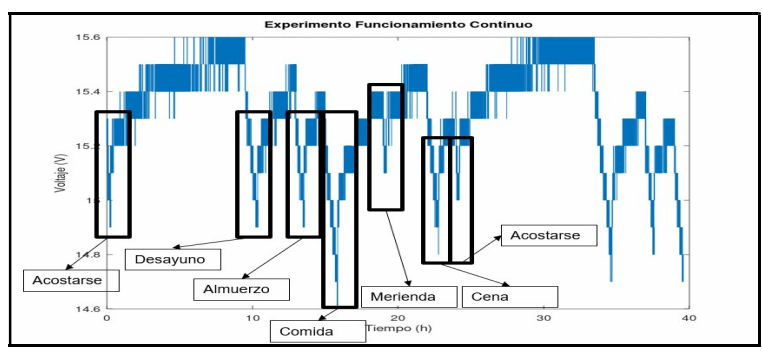

Figura 8: Curva típica de funcionamiento de 1 día (Tabla 3).

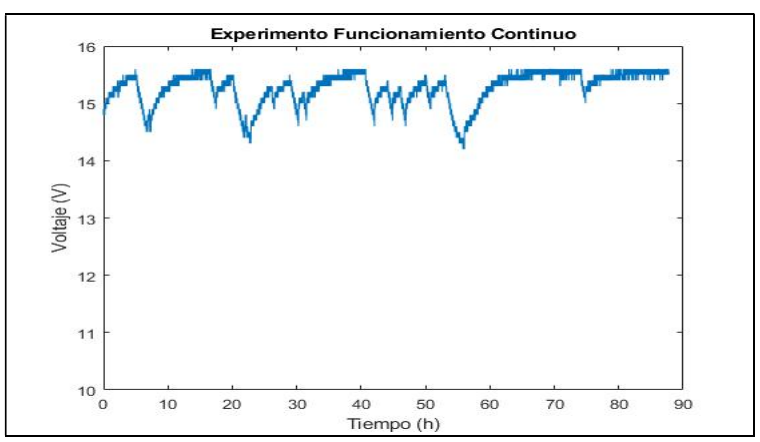

Figura 9: Experimento 1 de funcionamiento continuo según la Tabla 3 (Hora de inicio del experimento 14:30).

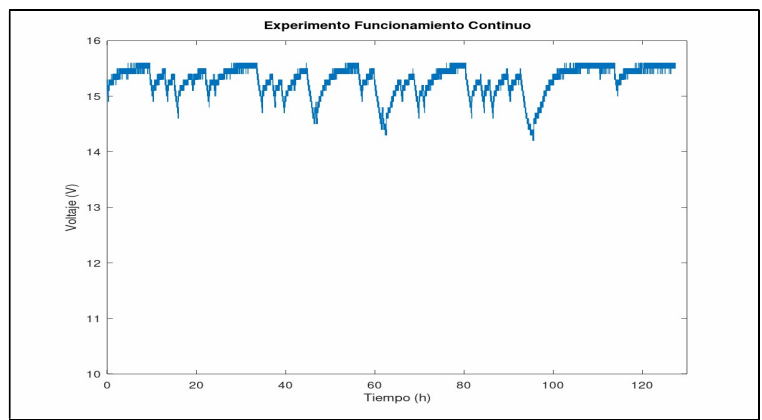

Figura 10: Experimento 1 de funcionamiento continuo según la Tabla 3 (Hora de inicio del experimento $23: 12$ ).

Como se puede observar en la Figura 9, se ven picos de carga y descarga de la batería debido a un funcionamiento continuo de 90 horas aproximadamente. Si se compara con la Figura 10, se verá que hay picos extraños que no concuerdan con el ciclo de 1 día. Esto se debía a la estación de carga del Turtlebot y al algoritmo de autodocking. Hay que tener en cuenta que, para llevar a cabo el proceso de autodocking, la estación de carga dispone de unos receptores infrarrojos mientras que la base del Turtlebot dispone de los emisores. Así pues, se llegó a la conclusión de que el sistema fallaba debido a que se encontraba en un sitio excesivamente luminoso y 
poco amplio. Para el siguiente experimento, mostrado en la Figura 10, se decidió poner la base en un lugar más oscuro y amplio. De esta manera se logró un funcionamiento más coherente con las especificaciones del dispositivo. Sin embargo, acabó fallando en algunos casos y se requirió de intervención manual.

Por tanto, se llegó a la conclusión de que el algoritmo de autodocking no era lo suficientemente fiable para una aplicación como la que se propone en este sistema. Actualmente se está trabajando en otros métodos alternativos mediante el uso de visión artificial. A pesar de este resultado, se puede concluir que el robot dispone de la suficiente autonomía para esta aplicación.

En cuanto a la parte de la aplicación Android y de la Empatica E4, se está aún en fase de prueba de la aplicación. De momento, se ha trabajado con cuatro sujetos de pruebas totalmente ajenos al proyecto que están ofreciendo realimentaciones de los posibles problemas de la aplicación.

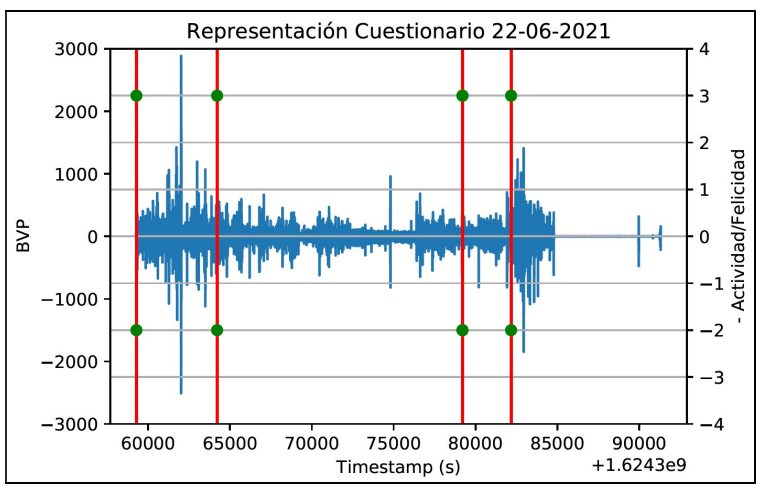

Figura 11: Representación de la señal BVP obtenida el 22-06-2021 con los test felicidad/actividad del día.

La Figura 11 es una representación de una de las variables obtenidas de la Empatica E4 junto con los cuestionarios del día 22-06-2021. De esta manera se pueden encontrar fallos más fácilmente y ver si son fruto de una mala lógica de programación o porque el usuario no ha cumplido con su responsabilidad. La señal azul se corresponde con el BVP, la recta vertical roja marca el timestamp en el que se ha respondido el cuestionario y los puntos verdes marcan la respuesta del usuario. Cabe destacar que para más claridad en los datos se ha decidido representar el valor negativo de la actividad. De esta manera el semieje positivo queda para la felicidad y el negativo para la actividad. Si se tiene en cuenta que debe haber 5 cuestionarios respondidos por cada día, se puede observar fácilmente que falta una línea roja. Tras analizar los datos se observa que el timestamp del tercer y cuarto cuestionario son muy cercanos. Esto quiere decir que el usuario no ha estado atento a la notificación del tercer cuestionario y lo ha hecho seguido al cuarto cuestionario. Por otra parte, con esta representación es fácil detectar posibles fallos con la Empatica E4. En la Figura 11 se puede ver que el tramo final de la señal de BVP es plano, lo que indica que el usuario, muy posiblemente, se haya quitado el dispositivo durante ese intervalo de tiempo.

En cuanto a la parte del sistema domótico, se ha conseguido montar con éxito un servidor Home Assistant de prueba con la puerta de enlace cc2531. Tras probarlo 15 días con los sensores Zigbee de Xiaomi se ha comprobado que no ha habido desconexiones, lo que permite deducir que el sistema es bastante estable. Cabe destacar que con la otra puerta genérica (Conbee II) no se ha conseguido dicha estabilidad (cada 6 horas había que reconectar manualmente algunos de los sensores, mayoritariamente los PIR).

En cuanto a los futuros trabajos, se centran en seguir desarrollando el sistema. Por la parte de la aplicación queda terminar de validar los datos para comenzar con la fase de entrenamiento del algoritmo de machine learning. En la parte de inteligencia artificial se van a usar algoritmos diferentes a los utilizados en [13] para mejorar la capacidad de predicción del estado de ánimo del sistema. Se continuará con la implementación de la arquitectura software del sistema para conseguir la interoperabilidad de todos los elementos que lo componen. Además, se debe realizar también el cambio de la estrategia de navegación autónoma usando navegación semántica y solucionar el problema del autodocking con otras estrategias como el uso de visión artificial. También se deberá buscar la forma de integrar Alexa 10 con ROS y programar una skill específicamente diseñada para el proyecto.

\section{Agradecimientos}

El proyecto HIMTAE / subproyecto Robwell (referencia RTI2018-095599-A-C22) ha sido financiado por el Programa Estatal de Investigación, Desarrollo e Innovación Orientada a los Retos de la Sociedad, en el marco del Plan Estatal de Investigación Científica y Técnica y de Innovación 2013-2016. Tiene una duración de 3 años, prorrogado hasta septiembre de 2022.

Está co-financiado por el programa Knut and Alice Wallenberg foundation WASP-AI de Suecia. 


\section{English summary}

\section{Heterogeneous System for Daily Activity Monitoring at Home and Well-being of Elderly People Abstract}

This paper presents the design of a heterogeneous system for the health care and well-being of elderly people living alone at home. The system consists of a mobile robotic device, a set of low-cost home automation sensors, a medical device such as an activity bracelet and an Android application for the study of user's mood. The system has been integrated using ROS, IoT (Internet of Things) technologies such as Node-RED and home automation technology such as Home-Assistant. This heterogeneous system is currently being developed in a national project RETOS.

Keywords: Assistive robotics, ageing, Ambient Assisted Living (AAL), Node-RED, IoT, interoperability, heterogeneous systems, ROS, Smart Home

\section{Referencias}

[1] EUROSTAT, "Mortality and life expectancy statistics," 2021. https://ec.europa.eu/eurostat/statisticsexplained/index.php?title=Mortality_and_life _expectancy_statistics.

[2] INE, "Encuesta Continua de Hogares (ECH)," 2021. https://www.ine.es/jaxi/Datos.htm?path=/t20/ p274/serie/prov/p02/10/\&file=02014.px\#!tabs -tabla.

[3] Samsung, "Samsung Bots, los compañeros del futuro," 2019. https://news.samsung.com/co/samsung-botlos-mejores-companeros-roboticos-encamino-a-enriquecer-la-calidad-de-vida-delas-personas.

[4] E. García, "Rassel, el robot que ayuda a las personas mayores," 2018. https://cadenaser.com/emisora/2018/12/07/ra dio valencia/1544174190 652009.html.

[5] M. Chan, H. Bocquet, E. Campo, T. Val, and J. Pous, "Alarm communication network to help carers of the elderly for safety purposes: A survey of a project," Int. J. Rehabil. Res., vol. 22, no. 2, 1999, doi: 10.1097/00004356199906000-00008.

[6] G. Demiris et al., "Nurse Participation in the design of user interfaces for a smart home system," in SMART HOMES AND BEYOND, 2006, vol. 19.

[7] L. Pigini et al., "Pilot Test of a New Personal Health System Integrating Environmental and Wearable Sensors for Telemonitoring and Care of Elderly People at Home (SMARTA Project)," Gerontology, vol. 63, no. 3, 2017, doi: 10.1159/000455168.

[8] A. P. Pentland, "Smart Rooms," Sci. Am., vol. 274, no. 4, 1996, doi: 10.1038/scientificamerican0496-68.

[9] E. Bellocchio, G. Costante, S. Cascianelli, P. Valigi, and T. A. Ciarfuglia, "SmartSEAL: A ros based home automation framework for heterogeneous devices interconnection in smart buildings," 2016, doi: 10.1109/ISC2.2016.7580798.

[10] T. Chakraborti, S. Srivastava, A. Pinto, and S. Kambhampati, "An ROS-based shared communication middleware for plug and play modular intelligent design of smart systems," arXiv. 2017.

[11] O. M. Mozos et al., "Stress detection using wearable physiological and sociometric sensors," Int. J. Neural Syst., vol. 27, no. 2, 2017, doi: 10.1142/S0129065716500416.

[12] F. T. Sun, C. Kuo, H. T. Cheng, S. Buthpitiya, P. Collins, and M. Griss, "Activity-aware mental stress detection using physiological sensors," in Lecture Notes of the Institute for Computer Sciences, SocialInformatics and Telecommunications Engineering, LNICST, 2012, vol. 76 LNICST, doi: 10.1007/978-3-642-29336$8 \_16$.

[13] D. Bautista-Salinas, J. R. Gonzalez, I. Mendez, and O. M. Mozos, "Monitoring and Prediction of Mood in Elderly People during Daily Life Activities," 2019, doi: 10.1109/EMBC.2019.8857847.

[14] Turtlebot, "TurtleBot2." https://www.turtlebot.com/turtlebot2/.

[15] Az-Delivery, "ESP32." https://www.azdelivery.de/es/products/esp32developmentboard. 